\title{
ENCAPSULATED THERMIONIC ENERGY CONVERTER WITH STIFFENED SUSPENSION
}

\author{
Jae Hyung Lee ${ }^{l}$, Igor Bargatin ${ }^{l}$, Kentaro Iwami ${ }^{1,2}$, Karl A. Littau ${ }^{l}$, Maxime Vincent ${ }^{3}$, \\ Roya Maboudian ${ }^{3}$, Z.-X. Shen ${ }^{1}$, Nicholas A. Melosh ${ }^{1}$, and Roger T. Howe ${ }^{I}$ \\ ${ }^{1}$ Stanford University, USA \\ ${ }^{2}$ Tokyo University of Agriculture and Technology, Japan \\ ${ }^{3}$ University of California at Berkeley, USA
}

\begin{abstract}
This paper reports a prototype of a mechanically and thermally robust encapsulated microfabricated thermionic energy converter ( $\mu$-TEC). We used conformal sidewall deposition of poly-SiC to form stiff suspension legs with U-shaped cross sections, which increase the out-of-plane rigidity and prevent contact with the substrate during the heating of the suspended emitter-a qualitative improvement over the previously reported planar SiC $\mu$-TECs [1]. Furthermore, we tested the new $\mu$-TECs with both barium and barium oxide coatings. The coatings reduced the work function of the $\mathrm{SiC}$ emitter to as low as $\sim 2.14 \mathrm{eV}$ and increased the thermionic current by 5-6 orders of magnitude, which is a key step toward realizing a highly efficient thermionic energy converter. In addition, we have sealed small arrays of $\mu$-TECs using an anodically bonded pyrex wafer.
\end{abstract}

\section{INTRODUCTION}

Thermionic energy converters (TECs) are unique heat engines that convert heat directly to electricity at very high temperatures, typically $>1000^{\circ} \mathrm{C}$. This energy conversion process is based on thermionic emission - the evaporation of electrons from conductors at high temperatures - and was first proposed as early as 1915. In the simplest case, the converter consists of two electrodes in the parallel-capacitor geometry and uses the thermionically emitted current to drive a useful load (Fig. 1). In the 1950s-1960s, the first practical TECs with conversion efficiencies of $10-15 \%$ were created and, in the subsequent two decades, both NASA and the Soviet space programs developed TECs as power sources for space missions [2,3].

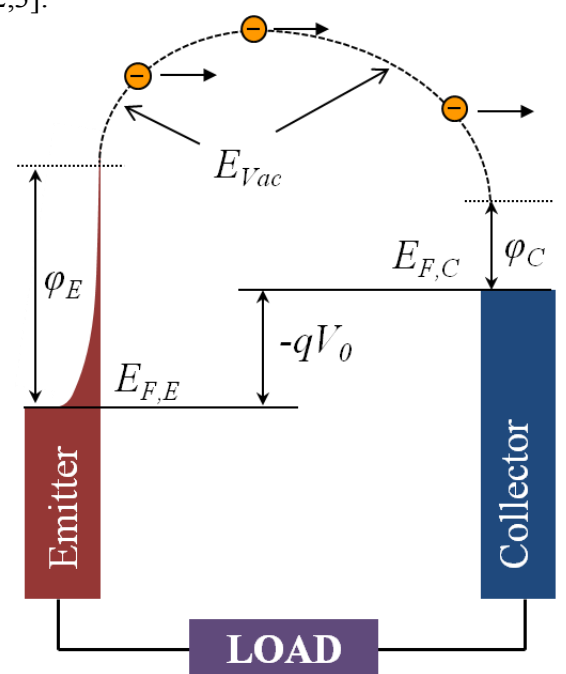

Figure 1: The energy diagram of the thermionic energy conversion process. $E_{F, E}$ and $E_{F, C}$ are the Fermi levels of the emitter and the collector, respectively. $E_{V a c}$ is the position-dependent vacuum level. $\varphi_{E}$ and $\varphi_{C}$ are the work functions of the emitter and the collector. $V_{0}$ is the voltage difference between two electrodes and $-q$ is the electron charge.
In the last two decades, major progress in microfabrication techniques prompted some people to reassess the possibility of making vacuum TECs with micron-scale gaps [3-5]. Microfabricated vacuum TECs are conceptually attractive because of their simpler physics. In addition, their efficiency limits can be very large fractions of the Carnot efficiency (Fig. 2) - exceeding solid state technologies (such as thermoelectric) and competitive with even conventional mechanical heat engines, such as steam turbines or Stirling engines.

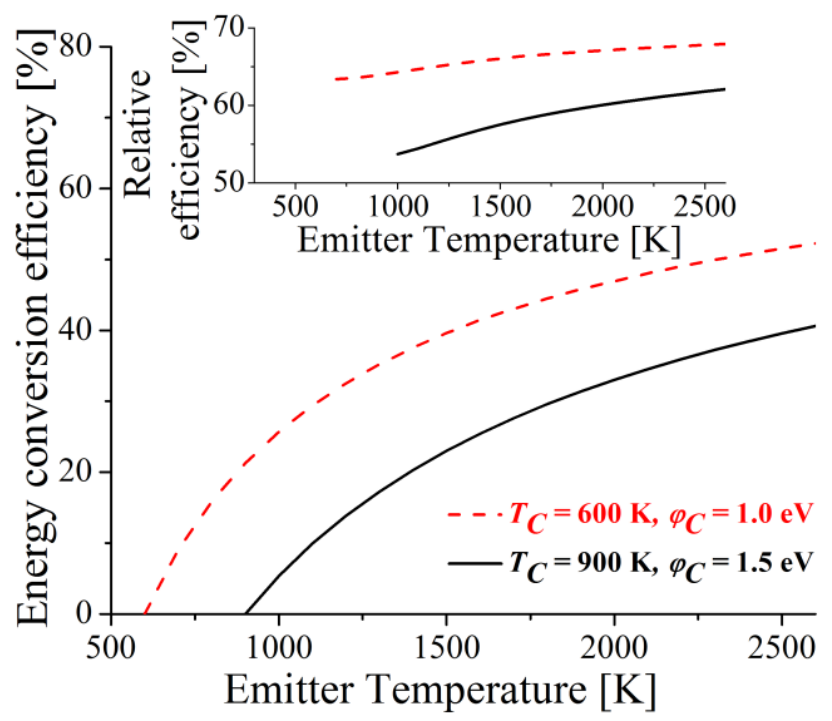

Figure 2: The maximum energy conversion efficiency of $\mu T E C$ versus the emitter temperature for collector work functions of 1.0 and $1.5 \mathrm{eV}$. We assumed the emitter work function proportional to the emitter temperature: $\varphi_{E}=T_{E}[K] / 750 \mathrm{eV}$, and the collector temperature of $T_{C}=\varphi_{C}[\mathrm{eV}] \times 600 \mathrm{~K}$. The inset shows the exergy efficiency, i.e., the energy conversion efficiency divided by the Carnot efficiency, $1-T_{C} / T_{E}$, for the corresponding emitter and collector temperatures. Based on calculations in Ref. [6].

We have recently developed a new approach to microfabricating TECs [1] that uses the substrate wafer as the collector and a plate suspended using flexible crablegs as an emitter (see inset of Fig. 3). Here we report various experiments with a new $\mu$-TECs design that uses stiff suspension legs, which increase the out-of-plane rigidity and help $\mu$-TECs to keep the gap in the optimal range during the operation.

\section{FABRICATION}

Images

Fig. 3 shows a photograph of an encapsulated die, which contains a parallel-connected $3 \times 3 \mu$-TEC array. The inset of Fig. 1 shows an SEM of an individual U-shape array element. Since all TECs were connected in parallel, only two contacts were needed to measure the I-V characteristic. The suspension width was $30 \mu \mathrm{m}$, 
the sidewall height was $30 \mu \mathrm{m}$, the emitter plate was $500 \mu \mathrm{m} \times 500 \mu \mathrm{m}$, and the poly-SiC film was $2-\mu \mathrm{m}$ thick. Etch holes were used in the emitter plate to facilitate the release. The gap between the poly-SiC emitter and the silicon substrate collector was approximately $10 \mu \mathrm{m}$.

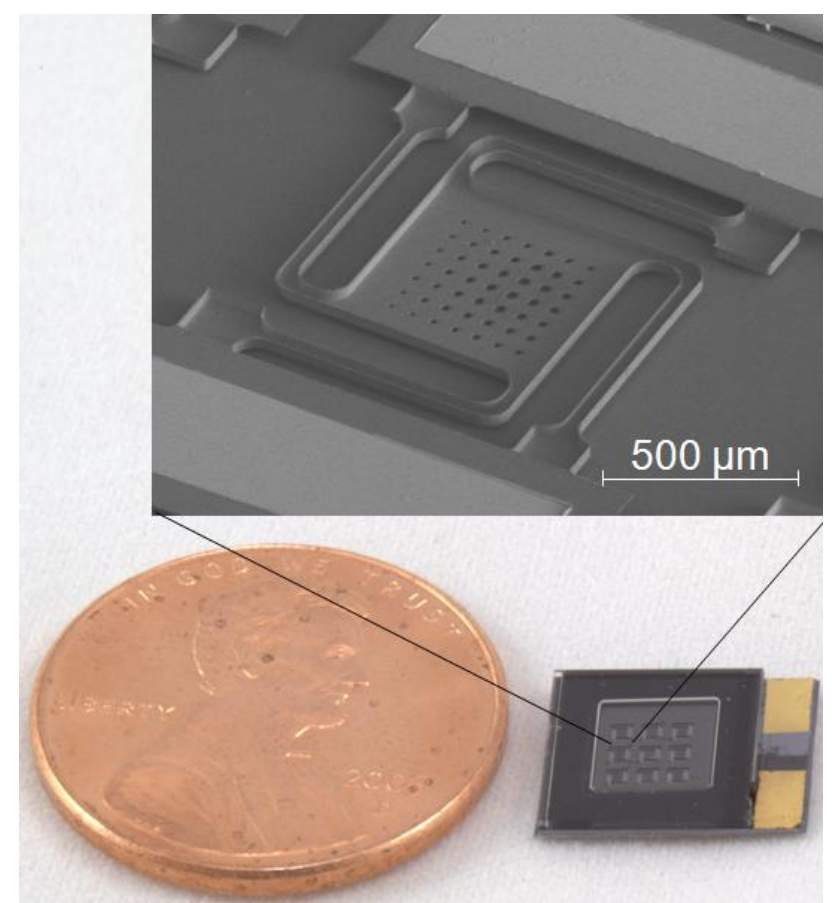

Figure 3: Vacuum-encapsulated die with a $3 \times 3$ array of TECs. A penny is shown to indicate scale. The inset shows a scanning electron micrograph (45 angle $\left.{ }^{\circ}\right)$ of one $U$-shaped TEC element.

Fabrication Process Flow



Figure 4: Outline of the fabrication process. (a) Initial 4-inch SOI

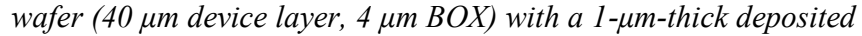
LTO hard mask. (b) After patterning LTO and DRIE etching to define 30-35 $\mu$ m deep silicon trenches. (c) After removing LTO layer and depositing 2- $\mu$ m-thick n-type polycrystalline 3C-SiC. (d) After patterning SiC layer using SPR 220-7 thick resist and anisotropic RIE. (e) After release using $\mathrm{XeF}_{2}$ and vapor HF etching. (f) After encapsulation and deposition of gold contact pads.
Fig. 4 outlines the fabrication process. The 4-inch SOI wafer was patterned to define $30-35 \mu \mathrm{m}$ deep silicon trenches before depositing polycrystalline $3 \mathrm{C}-\mathrm{SiC}$. The buried oxide layer of the SOI wafer was used to define the gap between the suspended emitter and the silicon substrate collector. After the release, the $\mu$-TECs were encapsulated using a 500- $\mu \mathrm{m}$-thick pyrex 7740 wafer with $250-\mu \mathrm{m}$-deep wet-etched cavities. The glass wafer was anodically bonded to encapsulate each $3 \times 3$ array, after which the wafer stack was diced and gold contact pads were deposited.

\section{EXPERIMENT \\ Optical Heating}

A single unencapsulated poly-SiC emitter was optically heated in a vacuum chamber by focusing the output of a blue laser diode (440-455 nm) onto a spot of $\sim 750 \mu \mathrm{m}$ diameter. The emitter was grounded at all times and the collector was biased to measure the current-voltage characteristic. Due to the increased out-of-plane rigidity of the U-shaped structures, we did not observe emitter-substrate shorting during optical heating, allowing us to make the emitter temperature higher than in our previous study [1].

Our $\mu$-TECs converted the estimated $270 \mathrm{~mW}$ of optical power incident on the emitter to $9 \mathrm{nW}$ of electrical power (Fig. 5), which is almost two-orders of magnitude higher than the power conversion efficiency in Ref. [1]. This still very low conversion efficiency $\left(<10^{-7}\right)$ is due primarily to the high work function of bare $\mathrm{SiC}$, which severely limits the thermionic current from the emitter [2]. Judging from the I-V characteristic, the work function of the silicon collector was even higher than that of the $\mathrm{SiC}$ emitter, further reducing the conversion efficiency.



Figure 5: The thermionic current versus the output voltage for different incident power to the SiC emitter. The thermionic current saturated when the output voltage was below $-1.5 \mathrm{~V}$. The inset shows that the maximum output power of this TEC is for an uncesiated $\mathrm{SiC}$ output voltage of $0.3 \mathrm{~V}$.

\section{Work function lowering by barium coatings}

Adsorbed cesium or barium can improve thermionic current from the emitter by reducing the work function of poly-SiC $[1,2,4,5]$. However, cesium reacts chemically with both silicon and silicon oxide, especially at elevated temperatures, which makes barium-based coatings easier to use in $\mu$-TECs made of standard silicon substrates. To investigate the effect of barium coatings on thermionic current, we measured the thermionic current both with 
and without barium coating on the $\mathrm{SiC}$ emitter in a small, dedicated vacuum chamber. After the pressure in the vacuum chamber reached $\sim 10^{-6}$ Torr, a commercial barium dispenser (Alvatec Production and Sales GesmbH) was heated resistively to evaporate the barium on the $\mathrm{SiC}$ emitter surface for a few minutes. Then, we resistively heated the $\mathrm{SiC}$ emitter while applying $40-80 \mathrm{~V}$ bias to the external metal collector, which was $\sim 1 \mathrm{~cm}$ away from the emitter, to collect most of the thermionic current from the emitter. The resulting reduction in the work function of the emitter enhanced thermionic currents from the heated $\mathrm{SiC}$ emitter by $5-6$ orders of magnitude (Fig. 6). At high temperatures ( $>1000 \mathrm{~K}$ ), the optical emissivity of polycrystalline $3 \mathrm{C}-\mathrm{SiC}$ is sufficiently large to enable temperature measurements using an optical pyrometer (PYRO MicroTherm, 550-nm operating wavelength). For emitter temperatures ranging from $900-1400 \mathrm{~K}$, we were able to fit our data to the Richardson-Dushman equation: $J=A_{\mathrm{E}} T_{\mathrm{E}}^{2} \exp \left(-\Phi_{\mathrm{E}} / k T\right)$, where $\Phi_{\mathrm{E}}$ is the emitter work function, $T_{\mathrm{E}}$ the emitter temperature, and $A_{\mathrm{E}}$ the materials-specific Richardson-Dushman constant. The work function of the barium-coated $\mathrm{SiC}$ obtained from the fit was approximately $2.14 \mathrm{eV}$ (the inset of Fig. 6).

We repeated the same experiment after we vented the vacuum chamber for a few hours and pumped the chamber back to $\sim 10^{-6}$ Torr to oxidize the barium coating on the $\mathrm{SiC}$ emitter. We observed approximately the same enhancement of thermionic current with the barium oxide coating (Fig. 6). However, the enhancement decreased irreversibly for both types of coatings if the emitter temperature exceeded the critical temperature of approximately $1600 \mathrm{~K}$. We believe that barium and barium oxide decomposed or desorbed from the $\mathrm{SiC}$ emitter above this temperature.

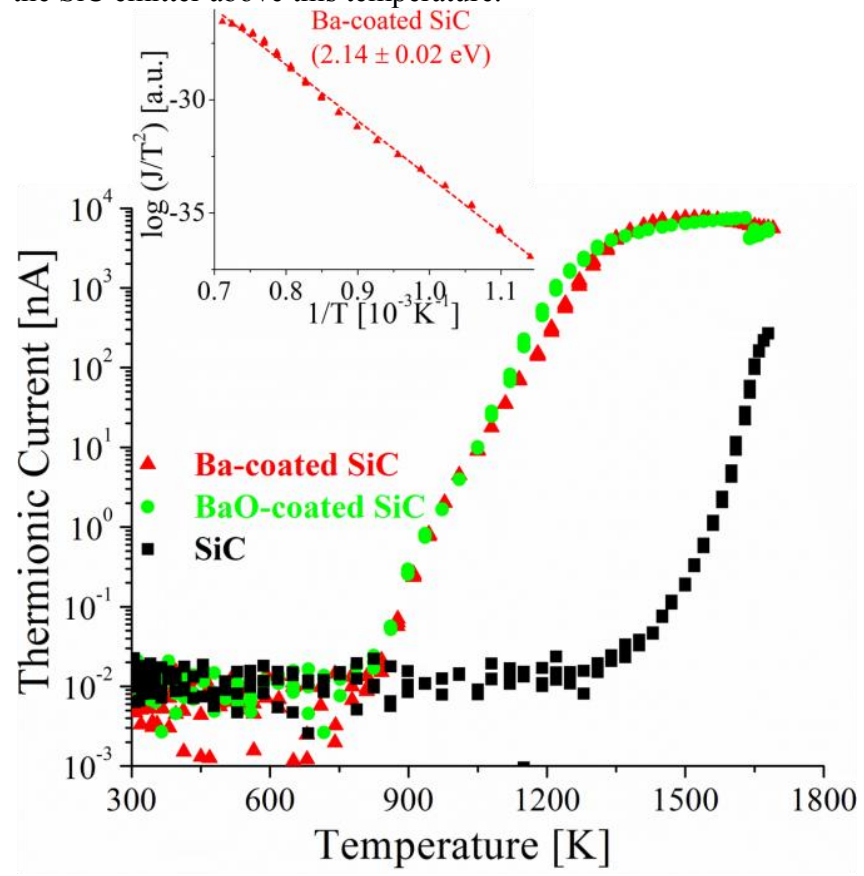

Figure 6: The thermionic current versus the temperature of the $\mathrm{SiC}$ emitter for both before barium coating, after barium coating, and after oxidizing barium which was coated on the SiC emitter. The inset shows the Richardson plot for the barium-coated SiC emitter.

To characterize the activation behavior of both barium coating and barium-oxide coatings, we measured the hysteresis of the thermionic current vs. temperature during upward and downward temperature sweeps (Fig. 6). Interestingly, a pure (unoxidized) barium coating exhibited much less hysteresis than a barium-oxide one, suggesting that pure barium coating does not need activation and can remain thermally stable in very high temperatures. It has been previously reported for barium-oxide coated emitters [7] that the barium atoms are released by the chemical reaction between the barium oxide and the substrate, and that these atoms migrate to the surface of the emitter coating by diffusion through the layer of barium oxide. The migration of barium atoms is facilitated by the high operating temperature of the emitter, and also by the porous, microcrystalline structure of the emitter coating $[7,8]$. This model may explain the difference in the hysteresis of pure barium and barium oxide coatings.

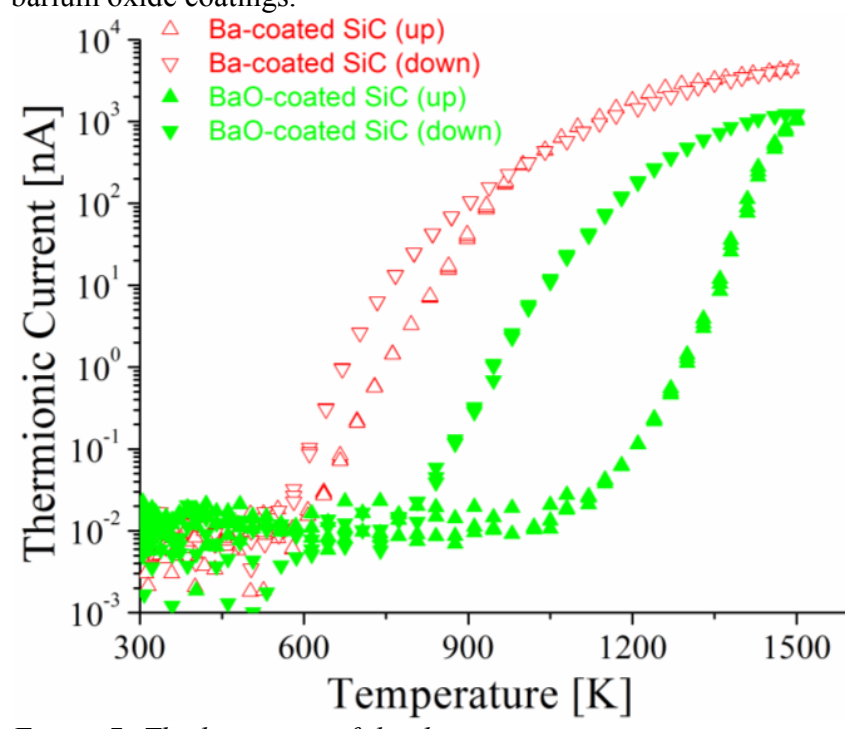

Figure 7: The hysteresis of the thermionic current vs. temperature for the barium and barium-oxide coated SiC emitter.

\section{$\boldsymbol{\mu}$-TEC Encapsulation}

Incorporating cesium into wafer-bonded vacuum packages has been previously demonstrated in the context of chip-scale atomic clocks [9]. $\mu$-TECs would require similar vacuum encapsulation with a work-function-lowering vapor. As an initial step toward achieving this goal, we have encapsulated small $\mu$-TEC arrays (Fig. 3).

We used the quality factor of the out-of-plane mechanical resonance of the suspended emitter to determine the residual gas pressure in the cavity. Toward that goal, we have measured the resonant response of the emitters in a vacuum chamber, which could be filled with a controlled pressure of nitrogen. The out-of-plane motion was actuated capacitively by applying $+3 \mathrm{~V}$ DC voltage to the collector and -15 to $5 \mathrm{dBm}$ of RF power to the emitter. A Polytec laser Doppler vibrometer was used to measure the out-of-plane motion of the suspended emitter inside the vacuum chamber at various pressures of nitrogen. Fig. 8 shows one example measured at a pressure of 0.2 Torr.

The quality factors of the detected mechanical resonance modes of the suspended emitter exhibit a strong dependence on the pressure of the ambient gas. Fig. 9 shows the quality factor of the fundamental resonance mode at $9.25 \mathrm{kHz}$ as a function of the nitrogen pressure. Using this curve, we will be able to calibrate the pressure of residual gas in vacuum encapsulated cavities.

While the bonding was successful, our initial experiments suggest that the packaging was not fully hermetic, and the cavity was eventually filled with ambient air to almost atmospheric pressure. (The quality factor of the encapsulated device was approximately 5.0). We plan to use an improved wafer bonding protocol to achieve hermetic vacuum encapsulation in the near future. 




Figure 8: Capacitively actuated out-of-plane resonance modes of the suspended emitter for the nitrogen pressure of 0.2 Torr. We obtained very good agreement between experiment and finite-element simulations: the experimental curve showed resonance frequencies at 9.25, 16.71, 20.08, and $39.25 \mathrm{kHz}$, whereas the COMSOL simulation predicted resonances at 9.55, $14.85,18.99$, and $37.07 \mathrm{kHz}$.

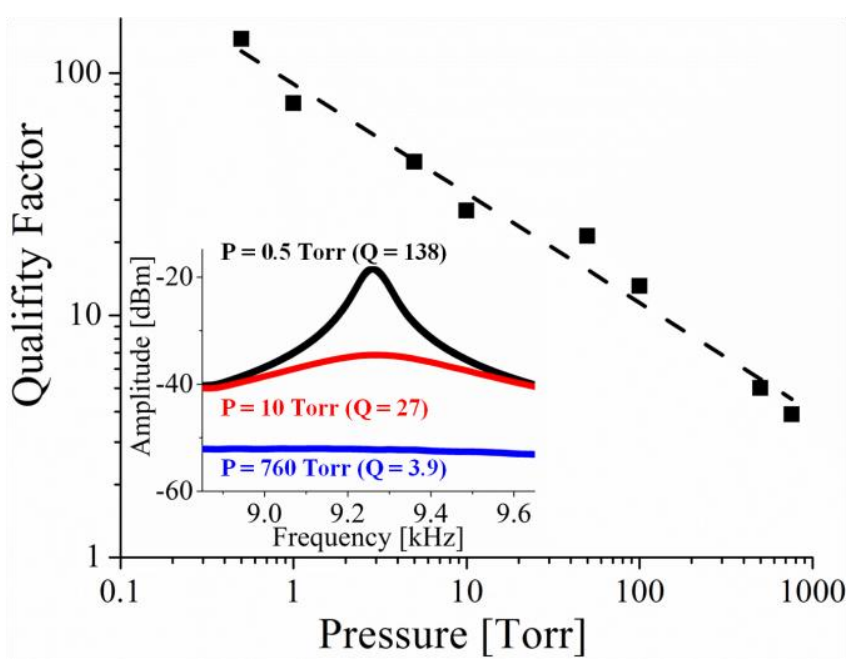

Figure 9: The quality factor versus the nitrogen pressure for the fundamental resonance mode at $9.25 \mathrm{kHz}$. The straight light is a guide to the eye. The inset shows representative resonance curves of the suspended SiC emitter for three different pressures.

\section{CONCLUSION}

We demonstrated a prototype of a mechanically and thermally robust encapsulated $\mu$-TEC. We used conformal sidewall deposition of poly-SiC to form stiff suspension legs with U-shaped cross sections, which increase out-of-plane rigidity and prevent contact with the substrate during the heating of the suspended emitter. We also showed that barium and barium-oxide coatings lowered the work function of the $\mathrm{SiC}$ emitter and enhanced thermionic currents from the heated $\mathrm{SiC}$ emitter by 5-6 orders of magnitude. We are currently working to demonstrate the high conversion efficiency of the $\mu$-TEC with barium or barium oxide coating of both electrodes.

We have thus achieved a number of milestones on the path towards realizing an efficient microfabricated thermionic energy converter. In the future, wafer-scale $\mu$-TECs can play an important role as topping cycles, adding to the efficiency of existing thermal energy converters such as thermoelectric or mechanical heat engines. Concentrated solar thermal power generation is a particularly attractive application for $\mu$-TECs due to the extremely high intrinsic temperature of solar radiation $(\sim 5900 \mathrm{~K})[10]$.

\section{ACKNOWLEDGEMENTS}

This work is supported by the Defense Advanced Research Projects Agency (DARPA) N/MEMS S\&T Fundamentals program under grant no. N66001-10-1-4004 issued by the Space and Naval Warfare Systems Center Pacific (SPAWAR), Robert Bosch North America, and OCI co. through the Center on Interfacial Engineering for MEMS (CIEMS), as well as BASF through the Stanford Energy and Environment Affiliates Program.

\section{REFERENCES}

[1] "Microfabricated Silicon Carbide Thermionic Energy converter for Solar Electricity Generation," J.-H. Lee, I. Bargatin et al., in Digest Tech. Papers MEMS Conference (2012)

[2] "Thermionic Energy Conversion Volume 1: Processes and Devices," G. N. Hatsopoulos and E. P. Gyftopoulos (Cambridge, MIT Press, 1973)

[3] "Thermionics: Quo Vadis", National Research Council, (National Academy Press, 2001)

[4] "Combustion-based Micro Power Generation: Thermoelectric and Thermionic Approaches," C. Zhang, PhD Dissertation, University of Michigan (2003)

[5] "Low Work Function Material Development for the Microminiature Thermionic Converter," D. King, K. Zavadil, D. Jennison, C. Battaile, A. Marshall, Sandia Report SAND2004-0555 (2004)

[6] "Optimal Emitter-Collector Gap for Thermionic Energy Converters," J.-H. Lee, I. Bargatin et al., Appl. Phys. Lett. (submitted, 2012)

[7] "Model of work function of tungsten cathodes with barium oxide coating," K. C. Mishra et al., J. Appl. Phys., 95, 3069 (2004)

[8] "Atomic and electronic structures of barium oxide on $\mathrm{Si}(001)$ studied by metastable impact electron spectroscopy (MIES) and coaxial impact collision ion scattering spectroscopy (CAICISS)," T. Ikeuchi et al., Appl. Surf. Science, 191, pp. 261-265 (2002)

[9] "Wafer-level filling of microfabricated atomic vapor cells based on thin-film deposition and photolysis of cesium azide," L. Liew et al., Appl. Phys. Lett. 90, 114106 (2007)

[10] "Photon enhanced thermionic emission for solar concentrator systems," J.W. Schwede, I. Bargatin et al., Nature Mater., 10, pp. 762-767 (2010) 